Radial and Nonradial Pulsations as Probes of Stellar Physics

ASP Conference Series, Vol. 259, 2002

C. Aerts, T.R. Bedding, \& J. Christensen-Dalsgaard, eds.

\title{
The Luminosities of $\delta$ Scuti Stars
}

\section{H. McNamara}

Department of Physics and Astronomy, Brigham Young University, Provo, Utah 84602, e-mail: mcnamarapasp@byu.edu

The $\delta$ Scuti stars are variables found in the instability strip above the zeroage main sequence. Radial and nonradial pulsation modes have been detected in these stars. The large-amplitude variables with asymmetric light curves are radial pulsators. The pulsation periods are found to be in the period range of $0.03-0.25$ days. Generally, the light amplitudes are small (total range $<0.3$ mag), but some fundamental-mode variables reach $0.70 \mathrm{mag}$. The majority of the variables have light amplitudes $<0.10 \mathrm{mag}$. Population II $\delta$ Scuti variables are frequently called SX Phe stars. Many of these variables have been found in globular clusters, where they populate the blue-straggler domain of the colormagnitude diagrams.

The most recent Period-Luminosity relation (P-L hereafter) is a linear relation appropriate for the fundamental pulsators, given by McNamara (1997):

$$
M_{v}=-3.725 \log P-1.933 \text {. }
$$

According to Petersen \& Christensen-Dalsgaard (1999), this equation should yield $M_{v}$ values to an accuracy of $\leq 0.1 \mathrm{mag}$.

We have re-examined the P-L relation by

- utilizing new evolutionary models and pulsation theory and

- utilizing HIPPARCOS parallaxes.

We find that in both cases a change in slope of the P-L relation is indicated at $\log P \sim-0.95$.

The new P-L relations are:

$$
\begin{aligned}
& \log P<-0.95 ; \quad M_{v}=-4.14( \pm 0.05) \log P-2.46( \pm 0.02), \sigma= \pm 0.16, \\
& \log P>-0.95 ; \quad M_{v}=-3.08( \pm 0.11) \log P-1.52( \pm 0.02), \sigma= \pm 0.14 .
\end{aligned}
$$

About 35 HIPPARCOS parallax stars have been employed in fixing the zero points of the equations. Lutz-Kelker corrections have been applied in deriving Eqs. (2) \& (3). Except near the transition region $\log P=-1.00$ to -0.80 , Eqs. (2) \& (3) give $M_{v}$ values similar to those given by Eq. (1) (within $0.1 \mathrm{mag}$ agreement).

We emphasize that $\delta$ Scuti variables (if pulsation modes can be identified) are capable of yielding $M_{v}$ values and hence distances of very high accuracy. The cosmic scatter around the P-L relations is $0.08 \mathrm{mag}$. This may be compared to 
the cosmic scatter of 0.24 mag of classical Cepheids about their P-L relation. They should be capable of yielding accurate distances out to and including Local Group galaxies. They have already proved useful in defining the luminosity levels of many features in globular clusters (McNamara, 2001).

\section{References}

McNamara, D. H. 1997, PASP, 109, 1221

McNamara, D. H. 2001, PASP, 113, 335

Petersen, J. O. \& Christensen-Dalsgaard, J. 1999, A\&A, 352, 547 